\title{
Autoimmune Diseases in Dermatology
}

JMAJ 47(9): 431-435, 2004

\author{
Hiroo YOKOZEKI* and Kiyoshi NISHIOKA** \\ *Associate Professor, **Professor, Tokyo Medical and Dental University
}

\begin{abstract}
This article summarizes the concept, clinical characteristics, and treatment of bullous diseases and erythema nodosum, which are typical autoimmune diseases in the field of dermatology. Autoimmune bullous diseases are classified into the pemphigus group, with antibodies against substances between epidermal cells, and the pemphigoid group, with antibodies against epidermal basement membrane. The causative antigens for each group have recently been identified. Type-III allergic reaction induced by bacteria, medicine, etc. as causative antigens is thought to be involved in the development of erythema nodosum. The treatment of the diseases chiefly consists of removal of causative antigens and steroid hormone therapy.
\end{abstract}

Key words: Pemphigus; Bullous pemphigoid; Erythema nodosum;

Autoimmune disease

\section{Autoimmune Bullous Diseases}

\section{Concept}

In autoimmune bullous diseases, antibodies against epidermal antigens damage the epidermis, resulting in bullous formation. Autoimmune bullous diseases are divided into two groups: the pemphigus group with antibodies against substances between epidermal cells, and the pemphigoid group with antibodies against epidermal basement membrane.

Desmosome and hemidesmosome are considered to play important roles in the adhesion of epidermal cells. Recent molecular-biological studies have shown that the antigens of all autoimmune bullous diseases are the components of desmosome and hemidesmosome. Figures 1 and 2 show the structure and component proteins of hemidesmosome and desmosome, respectively.

Desmosome exists in epidermal cell membrane. Keratin intermediate-sized filaments combine with the intracellular adhesion plate. Two membrane proteins of desmoglein and desmocollin maintain intercellular adhesion, each of which is subdivided into Types 1 to 3 . Intracellular adhesion is based on desmoplakin (DPL), envoplakin (EPL), periplakin (PPL), placoglobin (PG), and placophilin. Types 1 and 3 desmoglein are the target antigens of pemphigus. ${ }^{1-3)}$

Keratin intermediate-sized filaments also combine with the adhesion plate of hemidesmosome. Intracellular adhesion plate pro-

This article is a revised English version of a paper originally published in the Journal of the Japan Medical Association (Vol. 129, No. 7, 2003, pages 931-935). 
teins include BP230 and plectin that belong to the plakin family. Trans-membrane proteins include BP180 and $\alpha 6 \beta 4$ integrin. BP180 and BP230 are the target antigens of bullous pemphigoid. ${ }^{1,4}$

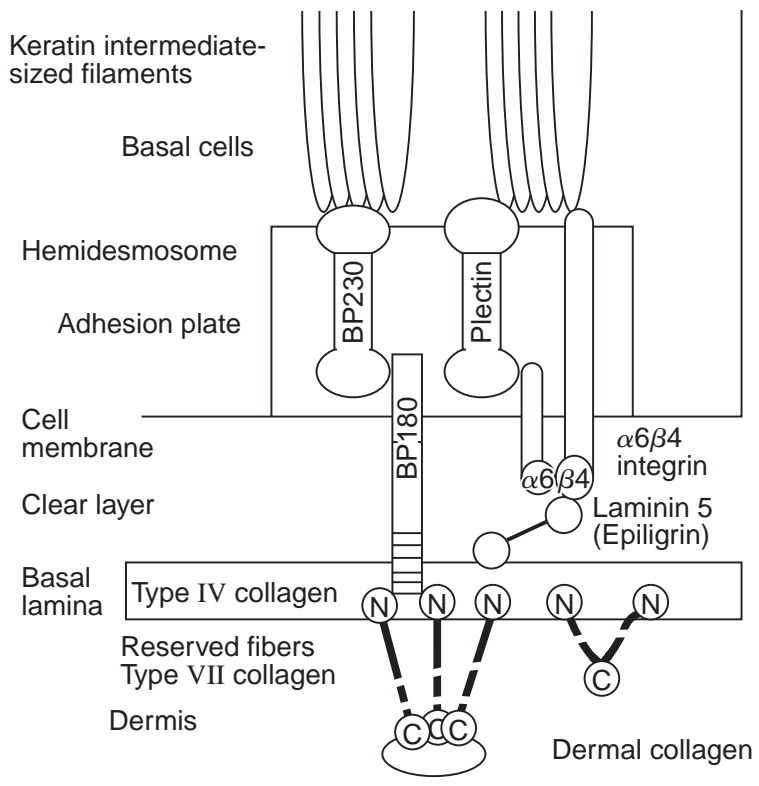

Fig. 1 Structure of hemidesmosome (see Reference 1)

\section{Pemphigus}

\section{(1) Definition and classification}

Pemphigus is a representative autoimmune dermal disease associated with autoantibodies against substances between epidermal cells. It is divided into four classic types - pemphigus vulgaris, pemphigus vegetans, pemphigus foliaceus, and pemphigus erythematosus - as well as other types of herpetiform, drug-induced, tumor-associated, and IgA pemphigus. The antigens of each type of pemphigus are listed in Table 1.

\section{(2) Clinical symptoms and pathological findings}

Pemphigus vulgaris often develops as intractable diffuse oral lesions, followed by loose bullae and erosions on the skin (Fig. 3). The bullae and erosions tend to develop at intertriginous sites and be epithelialized without scarring.

Pemphigus vulgaris is associated with the bullous formation on mechanically stimulated normal skin, which is called "Nikolsky's sign." Histopathologically, acantholysis (epidermal cells are separated from each other to form

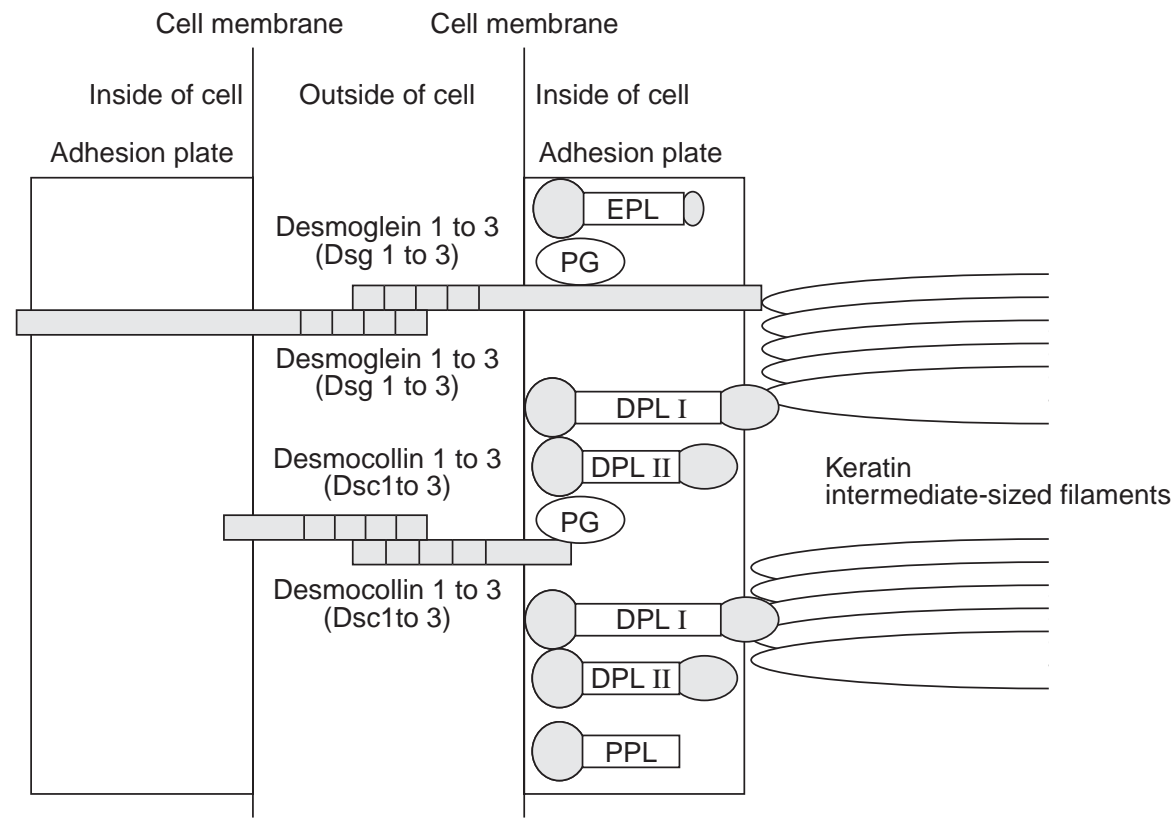

Fig. 2 Structure of desmosome (see Reference 1)

(EPL: Envoplakin, PG: Placoglobin, DPL: Desmoplakin, PPL: Periplakin) 
Table 1 Classification and Antigens of Pemphigus

\begin{tabular}{l|c|l}
\hline \hline \multicolumn{1}{c|}{ Subtype } & Immunoglobulin & \multicolumn{1}{c}{ Antigen } \\
\hline Pemphigus vulgaris & IgG & Desmoglein 3 (DG3) \\
\hline Pemphigus vegetans & $\operatorname{IgG}$ & DG3 \\
\hline Pemphigus foliaceus & $\operatorname{IgG}$ & DG1 \\
\hline Pemphigus erythematosus & IgG & DG1 \\
\hline Herpetiform pemphigus & IgG & DG1 (DG3) \\
\hline Drug-induced pemphigus & IgG & Various \\
\hline Tumor-associated pemphigus & IgG & $\begin{array}{l}\text { Desmoplakin, envoplakin, periplakin, BP230, } \\
\text { DG1 and 3, and 170 kDa protein }\end{array}$ \\
\hline IgA pemphigus & IgA & Desmocollin 1 \\
\hline
\end{tabular}

(Quoted from Hashimoto, T.: Hydroa. Easy Dermal Immunology (Nishioka, K. ed.) 2002; pp. 199-208) (in Japanese)

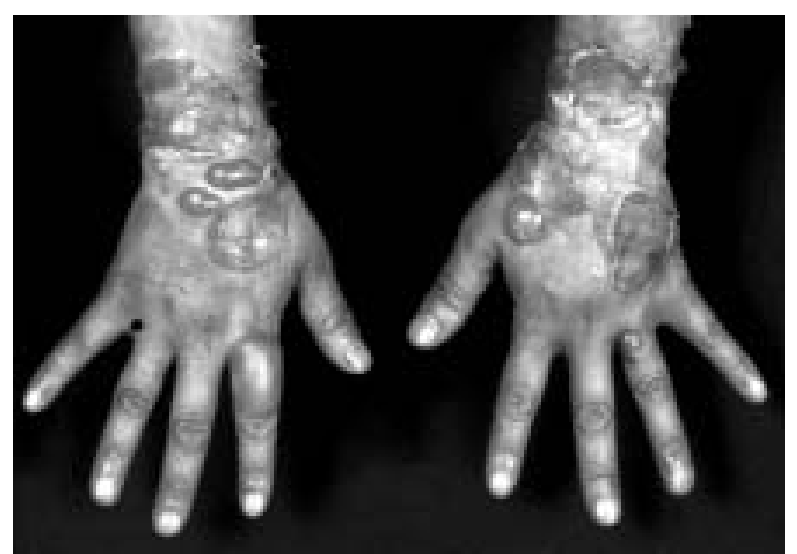

Fig. 3 Clinical case with pemphigus vulgaris

intraepidermal bullae) is observed over the basal layer. Skin biopsy using a fluorescent antibody technique shows the deposition of IgG and $\mathrm{C} 3$ between epidermal cells.

Pemphigus vegetans is a subtype of pemphigus vulgaris and appears as a verruciform skin elevation associated with epidermal hypertrophy by the long-term mechanical stimuli at intertriginous sites.

Pemphigus foliaceus causes shallow erosions, squamae, and crusts at seborrheic sites of the head, face, chest, and back. The erosions are quickly resolved, although pigmentation remains. This type of pemphigus is usually not associated with oral lesions.

Pemphigus erythematosus is a subtype of pemphigus foliaceus. It causes exanthema at seborrheic sites as in pemphigus foliaceus. Furthermore, it causes a butterfly erythema associated with keratose erosions on the face. It may be positive for antinuclear antibodies and complicated with thymoma. The biopsy of pemphigus foliaceus and erythematosus shows subcorneal bullae associated with acantholysis.

\section{(3) Diagnosis}

Pemphigus can be easily diagnosed based on clinical symptoms, histopathological examination, fluorescent antibody technique, and enzyme-linked immunosorbent assay (ELISA). It has to be differentiated from other autoimmune bullous diseases, drug eruption, and impetigo contagiosa.

\section{(4) Treatment}

Pemphigus vulgaris or vegetans should be treated with steroid hormones at an initial dose of 40 to $60 \mathrm{mg} /$ day (the dosage is based on predonisolone $^{\circledR}$ ). If the disease does not respond, the dose should be increased 1.5 to 2 times or steroid pulse therapy performed. Previous studies reported that the combination with immunosuppressive agents (such as cyclosporin and MTX), plasmapheresis, and massive $\gamma$-globulin therapy was effective for 
intractable pemphigus. Pemphigus foliaceus often requires a smaller oral dose of steroid hormone than pemphigus vulgaris: an initial dose of 20 to $40 \mathrm{mg} /$ day (based on predonisolone ${ }^{\circledR}$ ) may be enough.

\section{Bullous pemphigoid}

\section{(1) Concept and definition}

Bullous pemphigoid is an autoimmune bullous disease in which autoantibodies react with $230 \mathrm{kDa}$ and $180 \mathrm{kDa}$ pemphigoid antigens in hemidesmosome to cause subepidermal bullae. Tense and filled bullae on severely itching edematous erythema in the elderly clinically characterize the disease.

\section{(2) Clinical symptoms and pathologic findings}

As described above, bullous pemphigoid tends to develop in the elderly. At first, urticaria-like or exudative erythema associated with severe systemic itching develops, followed by the gradual formation of tense and filled large bullae on erythema. The bullae, when ruptured by scratching, cause erosions that look like burns on the whole body. Severe bullous pemphigoid may be complicated with fever, body weight loss, and anemia. Histopathologically, subepidermal bullae associated with eosinophilic and neutrophilic infiltration characterize the disease. Fluorescent antibody technique shows the linear deposition of $\mathrm{IgG}$ and $\mathrm{C} 3$ along the basement membrane.

\section{(3) Treatment and prognosis}

Mild bullous pemphigoid may be resolved only with the external application of steroid hormone. However, the disease is basically treated with the oral administration of steroid hormone: an initial dose of $40 \mathrm{mg} /$ day (based on predonisolone ${ }^{\circledR}$ ) is often used. Sulfa agents, such as diaminodiphenyl sulfone (DDS) and diaphenylsulfone $\left(\right.$ Lectisol $^{\circledR}$ ), or the combination therapy of minocycline and nicotinamide have been reported to be effective.

The prognosis of the disease is good, and the disease can be controlled with the oral administration of steroids. However, since many patients with the disease are elderly, attention should be paid to possible infections and malignant tumors.

\section{Erythema Nodosum}

\section{Concept}

Erythema nodosum is a painful erythema that develops on the extensor side of the leg. Histologically, septal panniculitis at the septum between subcutaneous fat and connective tissue characterizes the disease.

\section{Etiology}

Etiologic factors of erythema nodosum include infection with bacteria including hemolytic streptococcus, Mycobacterium tuberculosis, Mycobacterium leprae, and viruses, as well as drugs, Behçet's disease, sarcoidosis, and ulcerative colitis. Among them, the infection with hemolytic streptococcus, such as tonsillitis, is considered the most important factor. Although it has been suggested that Type III allergic reaction to these antigens may be involved in the development of the disease, this hypothesis has not been fully examined. Erythema nodosum is considered to occur in 10 to $15 \%$ of patients with Sweet's disease and $80 \%$ of those with Behçet's disease.

\section{Clinical symptoms}

Erythema tends to occur on the extensor side of the leg, although it may occur on the femoral region and forearm. Erythema appears as a painful and poorly defined mild elevation at which subcutaneous induration is palpable (Fig. 4). It is associated with heat, but not with erosions and ulcers. Erythema disappears in several weeks, but pigmentation remains. It is often associated with fever and may be associated with articular pain of the knee.

\section{Histopathological findings}

Making a diagnosis of erythema nodosum needs a skin biopsy to histopathologically examine the erythematous lesion. The lesion extends from the dermal substratum to sub- 


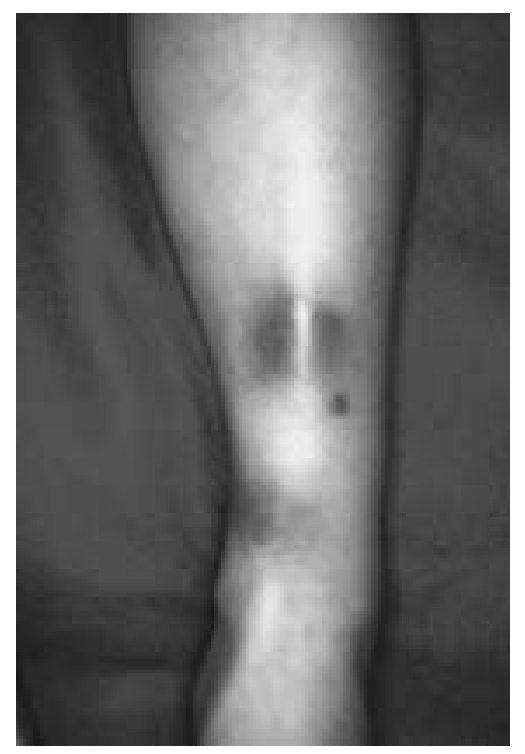

Fig. 4 Clinical case with erythema nodosum

cutaneous tissue and mainly results from septal panniculitis or inflammation of fat tissue at the septum with connective tissue. In the early stage, the disease shows neutrophilic and lymphocytic infiltration and fibrin precipitation in fat tissue. It then gradually presents chronic granulomatosis-like findings, such as infiltration of histiocytes and giant cells, hyperplasia of capillary vessels, and increase in fibroblasts.

\section{Laboratory findings}

Erythema nodosum is associated with inflammatory findings including increased white blood cell count, blood sedimentation rate and CRP. Increased ASO and ASLO are observed in patients infected with hemolytic streptococcus.

To examine the possible complication by pulmonary tuberculosis and sarcoidosis, it is also necessary to perform chest $\mathrm{X}$-rays and a tuberculin test.

\section{Diagnosis and differentiation}

Any painful erythema associated with subcutaneous induration on the leg has to be pathologically examined with skin biopsy to differentiate it from the following diseases.

\section{(1) Erythema induratum (Bazin)}

This type of erythema can be clinically differentiated by ulcerative erythema on the leg with persistent scars. Histopathologically, the diagnosis of the disease can be made with histological findings of lobular panniculitis, in which inflammation occurs mainly in fat tissue lobules, and epithelioid cell-like granuloma and caseation necrosis.

\section{(2) Thrombophlebitis}

Restiform induration clinically characterizes thrombophlebitis. This disease can be histopathologically differentiated from erythema nodosum.

\section{(3) Periarteritis nodosa (cutaneous type)}

Subcutaneous induration consistent with the crossings of livedo reticularis clinically characterizes the disease. Pathologically, this disease appears as necrotic angitis.

\section{Treatment}

Erythema nodosum improves only by elevating the leg and allowing the patient to rest. At first, an infectious focus of tonsillitis is located. When an infection with hemolytic streptococcus is suspected, it is necessary to administer a penicillin antibiotic. An underlying disease such as tuberculosis, fungal infection, sarcoidosis, or Sweet's disease, if any, is treated. Temporary systemic administration of steroid hormone is attempted in patients with severe fever and arthralgia without infectious focus and underlying disease, although this should be done carefully.

\section{REFERENCES}

1) Hashimoto, T.: Hydroa. Easy Dermal Immunology (Nishioka, K. ed.) 2002; pp.199-208.

2) Amagai, M. et al:: Autoantibodies against a novel epithelial cadherin in pemphigus vulgaris, a disease of cell adhesion. Cell 1991; 67: 869-877.

3) Amagai, M. et al.: Absorption of pathogenic autoantibodies by the extracellular domain of pemphigus vulgaris antigen (Dsg3) produced by baculovirus. J Clin Invest 1994; 94: 59-67.

4) Korman, N.J.: Bullous pemphigoid. Arch Dermatol 1998; 134: 1137-1141. 